\title{
Biomaterials at Materials Science and Engineering (MSE) 2012
}

$1 \quad$ Aldo R. Boccaccini Prof. Dr.-Ing. habil.

Head, Department of Materials Science and Engineering, Institute of Biomaterials, University of Erlangen-Nuremberg, Germany
2 Klaus D. Jandt PhD

FADM Chair of Materials Science, Otto Schott Institue of Materials Research (OSIM), Friedrich-Schiller-University Jena Löbdergraben, Jena, Germany
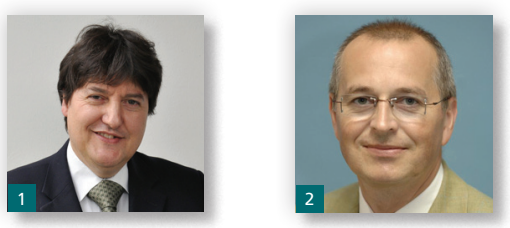

The Materials Science and Engineering (MSE) 2012 conference was held in Darmstadt, Germany, on September 25-27th 2012. This conference, organized under the auspices of the German Materials Society (DGM) included the topic 'Biomaterials' as one of the six key materials science and engineering areas represented at the conference. Within this topic, five symposia were organized on the following subjects: biomaterials applications, cell instructive and nanobiomaterials, bioinspiration and bioengineering, biointerfaces and coatings and materials for bone-tissue regeneration. These symposia attracted great interest with the presentation of more than 140 oral and poster contributions. The present special issue in Bioinspired, Biomimetic and Nanobiomaterials (BBN) includes articles authored by invited authors who presented their works at MSE 2012. There are a growing number of researchers in universities, research establishments and industry dedicating their research efforts to the ever expanding field of biomaterials. The challenges in the field continue to grow and the increasing significance of interdisciplinary research constitutes one of the attractive features of biomaterials science leading also to exciting opportunities to tackle persistent or complex medical problems with novel biomaterial based solutions. In addition, the related fields of bionics and biomimetics also featured with relevant symposia at MSE 2014, and are subjects covered by $B B N$.

The present special issue in $B B N$ features nine articles focusing on diverse aspects of the development, properties and applications of biomaterials covering a wide range of areas from surface functionalization strategies, cell-material interactions, nanobiotechnology, protein-based biomaterials, drug delivery devices, mineralization, biotemplating and biomimetic approaches.

The article by Berger and Fischer 'Photo-Induced Switchable $\mathrm{TiO}_{2}$ Thin Films for Biological Applications' considers advanced analytical methods to characterize functional groups and charge of titania films which were also exposed to fibroblasts to investigate the effect of the surface condition on cell growth.
In fact, understanding the effects of surface characteristics on cell behavior continues to be a major research topic in the biomaterials field, and their knowledge is key to developing strategies to improve the biological response of implant materials and tissue scaffolds. The article of Pang et al. 'Adhesion of Streptococcus mitis and Fusobacterium nucleatum on Nanopatterned Titanium Surfaces' discusses the adhesion of two oral bacteria on nanopatterned titanium surfaces aiming at understanding how specific welldefined nanoscale patterns (e.g. nanopillars of different heights and diameters) can be useful to control bacteria attachment on implants surfaces. Indeed, the relevance of the composition and topography of interfacial layers to impart specific cellular response is well known. The article of Skorb et al. 'Formation of Polypyrrole/ Metal Hybrid Interfacial Layer With Self-regulation Functions via Ultrasonication' presents a method to form polymer/metal hybrid interfacial layers with switchable properties which can have broad applications for example in self-healing approaches and for tailored hydrophilic surfaces. The subject of crystalline monolayers of polymer particles as templates for surface microstructuring is presented in the article by Bauer et al. 'Size and Shape Evolution of PS Particle Layers During Etching' where oxygen plasma technique is used to tune the particle geometry. The field of controlled and local drug delivery from novel biomaterials continues to attract increasing research interest. This field is represented in the present special issue by the article of Basnett et al. 'Aspirin-Loaded $\mathrm{P}(3 \mathrm{HO}) / \mathrm{P}(3 \mathrm{HB})$ Blend Films: Potential Materials for Biodegradable Drug-Eluting Stents' in which the authors demonstrate the convenient use of a biotechnology-derived biodegradable polymer as carrier for a model drug presenting a complete study of the drug-release ability and cytocompatibility of the system. Clearly, the development of advanced biomaterials and devices will consider increasingly biomimetic approaches and the application of more biocompatible natural materials. In the broad field of nanobiotechnology, protein-based materials continue to attract interest and represent the technology of choice to mimic nature's biological compartments. In this field, the 
review article of Schreiber and Schiller 'Nanobiotechnology of Protein-Based Compartments: Steps Toward Nanofactories' gives a comprehensive overview of synthetic biology approaches to generate compartment-forming protein-based modules, which will be relevant to the further development of the fields of nanofactories and nanoreactors, including the generation of suitable tools for 'genetic programming' functions. Certainly, genetic engineering of specific proteins is also an attractive approach to develop functionalized biotemplates for a wide range of applications. The article of Rothenstein et al. 'Mineralization of Gold Nanoparticles Using Tailored M13 Phages' shows an example of the development of biotemplates for the mineralization of inorganic nanoparticles. In particular, gold nanoparticles were mineralized locally by using gold-binding peptides as functionalized biotemplates. Biofunctionalization by specific immobilization of proteins on biomaterial surfaces is indeed an expanding field in biomaterials. Under controlled surface chemistry, the surface properties of polymers, metals and ceramics, in terms of protein adsorption and cell behavior, can be fundamentally altered. Biofunctionalization of 'standard' biomaterials has also important applications in other fields, notably in biotechnology. Successful research in this field considers biomimetic strategies to develop tailored functional surfaces. In this context, the article by Homaeigohar et al. 'Biofunctionalized Nanofibrous Membranes Mimicking Carnivorous Plants' presents a novel approach to immobilize specific protein ligands on the surface of polymer nanofibers, thus enhancing the selectivity of nanofibrous membranes. Indeed, understanding the structure-property function of natural materials continues to be a key challenge and an important research area attracting the effort of numerous researchers. Such knowledge provides a powerful tool for the design and development of new biomaterials structures. In this important field, the article by Zaslansky et al. 'Apatite Alignment and Orientation at the Angstrom and Nanometer Length Scales Shed Light on the Adaptation of Dentine to Whole Tooth Mechanical Function' provides evidence about the important effect of geometry and spatial arrangement of the mineral phase on tooth's mechanical behavior.

We hope that this special issue 'Biomaterials' of $B B N$ covering a range of topics relevant for the readers of this journal will contribute to enhance the knowledge in the interdisciplinary field of biomaterials and biomimetics and will stimulate further research and development in the field. 